\title{
De la caída relativa a la caída absoluta del salario real en la Argentina (1950-2013)
}

\author{
Juan Kornblihtt, ${ }^{1}$ Tamara Seiffer, ${ }^{2}$ Nicolás Villanova ${ }^{3}$ \\ Universidad de Buenos Aires (Argentina)
}

Recibido: agosto 25 de 2014 - Revisado: octubre 29 de 2014 - Aceptado: noviembre 12 de 2014

Referencia formato APA: Kornblihtt, J., Seiffer, T., \& Villanova, N. (2014). De la caída relativa a la caída absoluta del salario real en la Argentina (1950-2013). Revista Cientifica Guillermo de Ockham, 12(2), 41-50.

\section{Resumen}

El artículo analiza la evolución del salario real de la clase obrera argentina entre 1950 y la actualidad. En general, los análisis del salario en Argentina señalan a la dictadura militar, instaurada en 1976, como el punto de quiebre y el momento de persistente disminución. En este sentido, se abona la idea según la cual el cambio de modelo neoliberal sería el causante de esa baja. Sin embargo, como veremos en este artículo, en las décadas previas a la dictadura el salario real, aunque se mantiene, disminuyó en relación con los salarios reales de los países con los cuales compartía su evolución. Uno de los resultados principales refuerza los estudios que muestran la disminución del salario en relación con los países más ricos, como una de las fuentes de ganancia extraordinaria que compensa, junto a la renta de la tierra y la deuda externa, la baja competitividad del capital radicado en el país. Esta tendencia no se revierte durante la década del 2000, aunque haya una recuperación del salario.

Palabras clave: Salario real, fuerza de trabajo, explotación, Argentina

\section{From relative to absolute real wage decline in Argentina (1950-2013)}

\section{Abstract}

In this paper we study the Argentinean working class real wage evolution between 1950 and the present. In general, the mainstream analysis of the Argentinean wages point the Dictartorship (1976-1983) as the breakpoint in its tendency to grow and the beginning of a persistent fall. In this sense, they support the idea of the neoliberal model as causing the decrease in the working class living conditions. However, in this paper we show that even if the real wage did not fall before 1975 it was stagnate and fall in relation to the evolution of the countries with during the 30s and 40 s they share the evolution. One of the main results of our study support other Works that demonstrate that relative fall of real wage is source of extraordinary profits that compensate is sum with the land rent and the foreign debt the lack of competiveness of the capitals located in Argentina. We also show that this tendency does not end during the 2000 s, even there is a wage recovery.

Keywords: Real wage, Workforce, exploitation, Argentina

* Proyecto de investigación realizado con el apoyo del Centro de Estudios e Investigación en Ciencias Sociales (CEICS).

1. Doctor en Historia, investigador asistente del Conicet (IEALC-UBA /ICI-UNGS). Dirección postal: Condarco 90, Ciudad Autónoma de Buenos Aires, Argentina. E-mail: jkornblihtt@gmail.com

2. Doctora en Ciencias Sociales. Investigadora asistente del Conicet. E-mail: tamara_seiffer@yahoo.com.ar

3. Doctor en Historia, investigador del CEICS. E-mail: nicovillanova@yahoo.com.ar 


\section{Introducción}

En los últimos cuarenta años, se observa en la Argentina una importante transformación en las condiciones en las cuales se vende la fuerza de trabajo. El hecho más destacado es la caída del poder de compra del salario (aunque se mejore la diversidad de bienes que se pueden adquirir por los cambios tecnológicos). Desde 1974 hasta la actualidad, la caída del salario real es cercana al $40 \%$. De la mano, se consolida una fuerte fragmentación entre los obreros con la expansión del empleo no registrado (sin aportes) y la llamada tercerización. Se suma a esto un incremento de la población desocupada que, aun cuando no tenga un salario, su condición de vida está determinada por la misma relación en cuanto se define en torno al acceso a él.

Algunos autores señalan como punto de quiebre de ascenso salarial la década de 1970, en particular el momento de instauración del régimen dictatorial en 1976 (Altimir, Beccaria \& Gónzalez-Rozada, 2002). Se sostiene que el cambio del régimen de acumulación de capital, es decir, de un modelo económico basado en la producción a otro fundado en las finanzas, sería una de las causas de la baja salarial del conjunto de los trabajadores (Basualdo, 2006). Otros estudios que indagan la evolución salarial de largo plazo (entre 1950 y la actualidad) también abonan en este sentido (González, 2007; Marshall, 1981), por su parte, señala que la mejora del nivel de vida de las familias obreras durante la década de 1960 se debe a una mayor participación de la fuerza de trabajo familiar en el mercado y no sobre la base de un aumento de los salarios. Esto se diferenciaría de la década de 1940, momento en el cual el nivel de vida de los asalariados habría mejorado sobre la base del incremento del salario real.

El objetivo de este artículo es analizar la evolución del salario real entre la década de 1950 y la actualidad. Si bien se observa una caída en términos absolutos a partir de los años setenta, la disminución salarial de los trabajadores argentinos comienza a manifestarse a partir de la década de 1950 en términos relativos; es decir, comparando el nivel salarial con el obtenido por los trabajadores de otros países. Esta hipótesis de trabajo, a su vez, permite analizar la evolución salarial en relación con la productividad del capital radicado en Argentina frente a otros capitales. Antes de avanzar con el desarrollo de los argumentos y los resultados de esta investigación, conviene precisar algunos aspectos conceptuales y metodológicos.

\section{La conformación histórica del nivel salarial en Argentina}

La conformación de un mercado de venta de fuerza de trabajo libre es un proceso histórico que determina el carácter del salario actual y su tendencia. La constitución del capitalismo en nuestro país en el siglo XIX, tiene como base el desarrollo agrario. Ante una economía en expansión y una baja densidad poblacional, sumado al hecho de que la población indígena fue aniquilada en lugar de disciplinada, el abastecimiento provino de la inmigración, en su mayor parte europea. Se trató, sobre todo, de población rural expulsada frente al proceso de industrialización del Viejo Continente. La escasa disponibilidad de fuerza de trabajo y la competencia con otras zonas que buscaban absorber población, determinaron un mercado laboral con niveles de ingreso altos en relación con Europa y el resto de América Latina, aunque por debajo de los EE. UU. y Canadá (Williamson, 1992). Esto marcaría ciertas características generales del salario que situaran a los obreros argentinos con ingresos por encima de la media del resto del continente y de otros países de inserción tardía en el capitalismo. No basta, sin embargo, con esta génesis para explicar el devenir posterior.

Las primeras décadas del siglo XX muestran un nivel salarial alto que se acerca al de los EE. UU. o Inglaterra, pero con grandes oscilaciones marcadas por altibajos muy profundos. Hacia finales de la década de 1910 y principios de 1920, estos altos niveles salariales alcanzados comienzan a deteriorarse, lo que da comienzo a un ciclo de conflictividad. Se produce una recuperación con la expansión económica previa a la crisis del treinta, motorizada por un breve pero fuerte ascenso de la renta diferencial de la tierra agrícola, para luego volver a caer a niveles bajos. En 1943, impulsadas por movilizaciones obreras que dieron fruto de la mano de las políticas desarrolladas por Perón desde la Secretaría de Trabajo y luego desde su gobierno, el poder de compra de los asalariados se elevaría hasta alcanzar, en términos de su poder adquisitivo, los niveles históricamente más altos y marcando los siguientes treinta años.

¿Qué permitió este incremento? En primer lugar, una fuerte expansión de la actividad industrial, sustentada en un incremento en la renta diferencial de la tierra gracias al boom de demanda y precios agropecuarios durante la Segunda Guerra Mundial y los primeros años de la posguerra. En segundo lugar, la producción de materias primas, al llevarse a cabo sobre condiciones no reproducibles (la tierra) mejores en relación con otros países en cuanto a fertilidad, clima y cercanía a puertos,

$42<$ Universidad de San Buenaventura, Cali - Colombia 
arroja una ganancia extraordinaria de forma permanente. Tercero, un ingreso que permite cubrir las ganancias de los capitalistas agrarios, pero, además, deja un plus que corresponde a los propietarios de la tierra. Los terratenientes reciben una parte de la riqueza total pese a no aportar ni capital ni trabajo, solo el monopolio de algo no producido. Por esta razón, a lo largo de la historia el resto de los capitales, a través de la mediación estatal sin distinción de gobiernos, les sacó parte de esa renta (Iñigo-Carrera, 2007; Sartelli, 2008). Dichas ganancias, cuyo destino supuesto son las manos de los dueños de la tierra como pago por su uso, se les escapa a través de impuestos específicos, el tipo de cambio y la intervención del comercio exterior.

Estas mediaciones también afectan al salario, ya que además de expandir la demanda de empleo por el sostén de industrias ineficientes, genera el abaratamiento de las mercancías producidas en el agro, en relación con los precios internacionales. Como resultado, el consumo de los obreros se amplía sin necesidad de que el capitalista pague más (Ińigo-Carrera, 2007).

El nivel salarial alcanzado durante el primer gobierno de Perón, marcará el techo del poder de compra del salario de los obreros argentinos. El salario real oscilará en torno a ese nivel en las siguientes décadas. Ante la reducción de las transferencias por renta de la tierra por la caída de los precios de las mercancías agropecuarias a partir de 1950, los empresarios intentarán la baja salarial, pretensiones que si bien estuvieron marcadas por una fuerte conflictividad que comienza en el segundo gobierno de Perón, no lograron una caída fuerte del salario, pero sí un retraso en su crecimiento. Las oscilaciones permanentes del salario real en torno al mismo nivel durante el periodo, contrastan con el alza en de precios en los EE. UU. y Europa durante el llamado boom de posguerra. Por esta razón el poder de compra de los obreros argentinos empieza a retroceder en forma sostenida en relación con sus pares de otros países y a convertirse en fuente de ganancia extraordinaria para los capitalistas radicados en el país. Sin embargo, para este período todavía no se expresa en una gran pérdida absoluta del nivel de vida alcanzado durante el peronismo, algo que empezará a verse a partir de 1975.

\section{Método}

El salario real es la capacidad de compra en relación con una canasta de bienes constante a lo largo del tiempo. Se diferencia del salario nominal en cuanto este expresa las variaciones de bolsillo sin tener en cuenta la inflación. A su vez, difiere del costo laboral que pagan los empresarios por emplear fuerza de trabajo, en particular aquello que corresponde al salario "indirecto" (jubilación, aportes patronales, obra social, etc.). El análisis del salario real es una de las tantas formas de aproximarse al valor de la fuerza de trabajo, entendida como una mercancía utilizada por el capital.

La medición del salario real para el conjunto de la economía fue tomada de Kennedy (2012). El autor utiliza como fuentes las cuentas nacionales y los informes de la Cepal (para reconstruir el salario del total de la economía entre los ańos 1935-1974 y 1980-1987) tanto como la encuesta permanente de hogares para el período posterior hasta el año 2010. Para los últimos años, hemos actualizado la evolución del salario con cifras de variación salarial del Indec. El deflactor utilizado para convertir el salario nominal en real (es decir, en poder de compra), es el índice de precios del consumidor (IPC) de la provincia de San Luis, por ser el que mejor ajusta a la evolución del IPC del Indec con el cual se construyeron las series históricas de inflación antes de la manipulación de los datos producidos por dicho organismo. ${ }^{4}$

Por otra parte, la evolución del salario industrial registrado privado en Argentina en relación al de los Estados Unidos, fue tomada de Ińigo-Carrera (2007) y luego deflactada con el PPP (Purchasing Power Parities) del ańo 2005. Se trata de una canasta de bienes y productos construida internacionalmente, que permite comparar el poder de compra de los salarios de obreros entre diferentes países. ${ }^{5} \mathrm{~A}$ su vez, hemos reconstruido el salario en dólares que rige en la industria manufacturera de varios países, con base en U.S. Bureau of Labor Statistics. Finalmente, se utilizaron las cuentas nacionales de Argentina para reconstruir la evolución del salario real del total de la economía y de la industria para los trabajadores registrados y no registrados, entre los ańos 1993 y 2007.

4. El IPC del INDEC para el Gran Buenos Aires es denunciado desde el año 2007 por la intervención del gobierno nacional. Por ello, los estudios sobre economía tienden a utilizar índices de precios alternativos denominados "siete provincias" (que es el resultado del promedio de los IPC de siete provincias que no han sido intervenidas), así como también el IPC de San Luis, el cual sigue una evolución semejante al del GBA del Indec hasta el año 2007 y luego tiende a mostrar un porcentaje mayor de precios de productos en relación al IPC Indec.

5. Para más detalle ver, Banco Mundial: Global Purchasing Power Parities and Real Expenditures. 2005. International Comparison Program. Disponible en: http://siteresources.worldbank.org/ICPINT/Resources/icp-final.pdf 


\section{Resultados discutidos}

\section{De la caída relativa a la caída absoluta (1975 a 2001)}

En general, se reconoce que la dictadura militar en Argentina, iniciada en 1976, marcaría un quiebre en la evolución del salario real en relación con las décadas previas. Más todavía si la comparación es con el año de 1974, cuando en un contexto excepcional marcado por el boom de materias primas previo a la crisis mundial, se alcanza un pico en términos de poder de compra de los asalariados cercano al primer gobierno de Perón (19461952). Sin embargo, el alto nivel salarial alcanzado se encontraba estancado desde el primer gobierno de Perón y había comenzado a perder capacidad de compra en relación con aquellos países que, hasta entonces, mostraban una evolución salarial similar. A partir del "rodrigazo" en 1975 y el golpe militar encabezado por Jorge Rafael Videla en 1976, el quiebre, aunque importante, no marcaría una tendencia novedosa, sino que profundizaría una perspectiva ya instalada (ver Figura 1).

\section{Figura 1}

Índice de salario para el conjunto de la economía argentina: 1935-2013

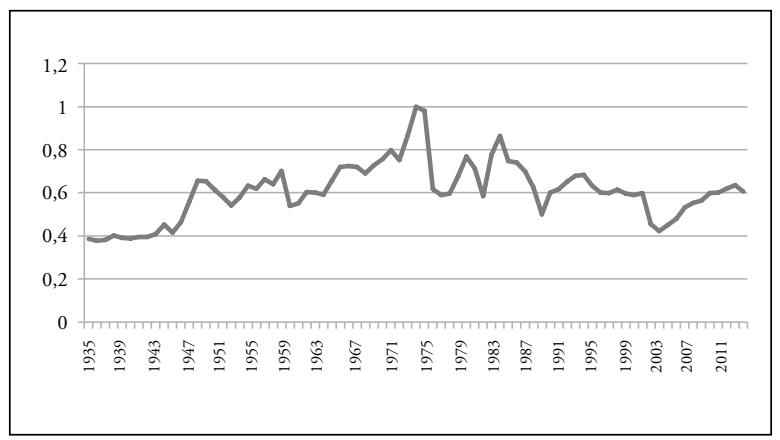

Fuente: Kennedy (2012), actualizado a partir de 2010, con datos del IVS-Indec e IPC San Luis.

Las explicaciones dominantes sobre las razones de la baja salarial a partir de mediados de los setenta, se centran en la idea de que a partir de la dictadura se instala un modelo neoliberal en el cual priman las finanzas por sobre la producción industrial (Basualdo, 2006; 2009; González, 2007). Según estos desarrollos, en el periodo previo primaba una pugna por sostener el mercado interno que llevaba a la generación de una comunidad de intereses entre la burguesía mercado-internista y los asalariados, comunidad que se expresaba en altos salarios que permitían cerrar la brecha entre demanda y consumo (ver Figura 2).

A estos intereses, se opondrían los de quienes priorizan el mercado externo y habrían triunfado a partir de 1975: los sectores exportadores radicados en el país (grandes
Figura 2 Salario industrial privado registrado de Argentina en relación con EE. UU, 1882-2008. $\mathrm{ppa}=2005$

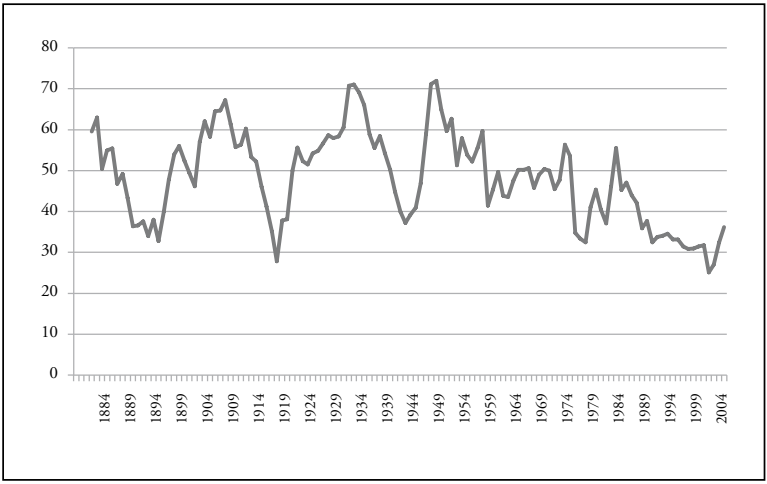

Fuente: elaboración propia con base en Iñigo-Carrera (2007) y el Banco Mundial.

empresas nacionales y extranjeras, especialmente los sectores vinculados al agro) y el sector financiero. Su triunfo explicaría la destrucción del mercado interno y un ataque a las condiciones salariales para, a partir de una política regresiva de distribución del ingreso, estimular las transferencias hacia el sector financiero y abaratar los costos para exportar (para una crítica a la explicación de Basualdo sobre el papel desempeñado por la baja salarial en una supuesta etapa de valorización financiera ver Fitzsimons, 2013).

Esta posición plantea una supuesta conciliación entre los intereses de los capitales más chicos (los mercado-internistas) y de los asalariados. Sin embargo y como muestran estudios específicos, los capitales argentinos más chicos pagan peores salarios, ya que de esa forma compensan su menor competitividad (Graña \& Kennedy, 2007; Graña, 2013). A su vez, estas teorías no contemplan el hecho de que el salario no surge de un reparto de la riqueza posterior a la generación de la ganancia, sino que es condición para su generación. El salario expresa las mercancías que debe consumir el asalariado para reproducir su capacidad de trabajar y la reproducción de sus hijos en cuantos futuros obreros, pero se trata de la única mercancía que tiene la particularidad de agregar más valor que su costo. El pago de ese salario normal se constituye en condición para la apropiación de la ganancia por parte del capitalista, y no a posteriori como producto de un correlato político en función de la distribución del ingreso (Marx, 1999). Si, además, el pago no alcanza el valor de la fuerza de trabajo, sería una fuente expandida de ganancia para los capitales en relación con los competidores que sí pagan por su valor. Dichas ganancias extraordinarias se obtienen a costa de atacar la propia vida de los trabajadores.

$44<$ Universidad de San Buenaventura, Cali - Colombia 
El salario estaría determinado por las características de los capitales que empleen esa fuerza de trabajo, en cuanto definen las características según el uso que le den y la magnitud de su demanda. En el periodo previo a 1920, la demanda de fuerza de trabajo se caracterizó, en un primer momento, por la necesidad de atraer inmigración en un contexto de escasez de asalariados. Un siguiente momento está marcado por la expansión de la industria mercado-internista, sostenida gracias a la transferencia de la renta de la tierra durante el gobierno peronista. Pero la fuente de transferencia de estos subsidios y protección, empezó a achicarse en función de la magnitud del capital que se acumulaba en el país. Según datos de Ińigo-Carrera (2007), representaba el $53 \%$ promedio de la plusvalía total neta de 1945 a 1955, mientras que de 1956 al 2009 significó apenas el $13 \%$. Frente a esta pérdida de la renta, los capitalistas buscaron aumentar sus ganancias a costa de los obreros. Los salarios argentinos perdieron, primero, en relación a lo que se expresaba en las necesidades de los trabajadores en los países donde la acumulación de capital es mayor; luego, en relación con el nivel alcanzado durante el peronismo. De las dos formas, los capitales que operan en el país comenzaron a obtener una ganancia extraordinaria que les permitió compensar (en parte) la caída de la renta diferencial de la tierra.

Esta baja salarial, sin embargo, no generó un aumento de la competitividad en términos internacionales. La baja productividad del trabajo de la industria argentina alcanza, en promedio, apenas el $19 \%$ de la de EE. UU. desde el año 1945 (Iñigo-Carrera, 2007) y no fue compensada con costos laborales competitivos.

Aunque en términos relativos a su propia historia y a la evolución en los países más potentes, el salario real cae a niveles muy bajos, cuando la comparación se hace frente a los países del este asiático o incluso al propio Brasil, el resultado es diferente. El empeoramiento de las condiciones de vida producto de la baja salarial, no alcanza para poner el costo laboral en dólares al nivel de los competidores que dominan las exportaciones en el mundo.

Esos países comienzan a atraer las inversiones de capital y con esa oferta de bajos salarios se da un desarrollo basado en dos tipos de capitales. Por un lado, pequeńos capitales con mucha mano de obra y poca mecanización (la confección, como rama paradigmática) y, por el otro, empresas de mayor composición orgánica, como por ejemplo, la siderurgia o la automotriz. La oferta de esa mano de obra barata es posible gracias a la existencia de una población rural mucho más densa que la existente en Argentina, dada la estructura agraria basada en la producción de arroz con base en sistemas de riego centralizados que no existían en Argentina. Este proceso histórico arroja una densidad de población disponible mucho mayor, la cual, junto a la fuerte represión política, crea las condiciones para la existencia de una clase obrera con niveles de vida muy bajos.

Ese tipo de inserción permite el desarrollo de una escala de producción más elevada que aumenta la demanda de fuerza de trabajo y complejiza el tipo de trabajo realizado. Por tal razón, a partir de la década de 1970 se observa en muchos de estos países una tendencia contraria a la de Argentina: desde puntos de partida de salario real mucho más bajos, se empieza a crecer. En algunos países o ramas de la producción, los salarios alcanzan, incluso, a superar el nivel de compra de los argentinos, lo que llevó a muchos capitales a trasladarse hacia países de la misma región con menor nivel salarial (por ejemplo de Corea del Sur a China y luego de China a Vietnam).

Figura 3

Salarios de la manufactura en dólares de paridad (2005), 1980-2010

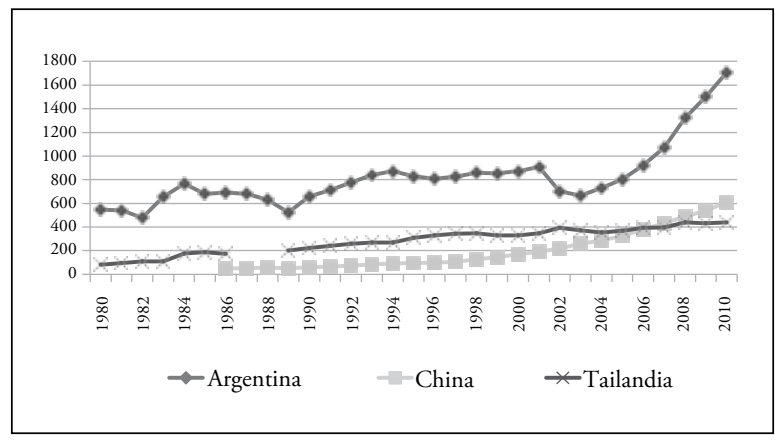

Fuente: Elaboración propia basada en U.S. Bureau of Labor Statistics.

El desarrollo de los salarios desde niveles muy bajos, pero con tendencia creciente que contrasta con la tendencia a la caída de los salarios argentinos, fue atribuido por algunos autores de tipo liberal a una supuesta falta de adaptación del nivel salarial a la productividad. Según estas teorías, el nivel salarial argentino está por encima de la productividad y esta sería el determinante del nivel de ingreso correcto, lo cual resulta incorrecto. La comparación de los salarios industriales con la productividad muestra que la segunda crece más rápido que el primero: mientras que entre 1976 y la actualidad los salarios industriales reales crecieron el $55 \%$, la productividad del trabajo aumentó el 328 \% (cálculo realizado según Ińigo-Carrera, 2007).

Sobre esta base falsa, los liberales proponen una fuerte baja salarial como forma de atraer capitales extranjeros, para luego repetir la experiencia-supuestamente virtuosadel sudeste asiático. Este planteamiento, que de alguna 
manera elogia la baja salarial implementada a partir de la dictadura, no toma en cuenta que los niveles a los que se debe llegar para competir con esos países son muy bajos. Asimismo, además de implicar un nivel de represión a la clase obrera incluso mayor que el ocurrido con el golpe de 1976, requeriría una densidad de población que no existe en Argentina.

En síntesis, lo que determina la baja salarial producida a partir de la dictadura, es la necesidad de aumentar la rentabilidad del capital, el cual ante su imposibilidad de expandirse por la vía de competir en el mercado externo, utiliza los menores costos laborales como una forma de compensar su ineficiencia, papel similar al que desempeñan la renta de la tierra y la deuda externa. Por esta razón, la tendencia decreciente del salario real en Argentina no es algo por completo novedoso -ya que rige desde el año 1975- ni se trata de adecuar los salarios a productividad, ni de un sacrificio para luego garantizar una mejora posterior.

El ciclo de baja del salario real abierto en 1975, adopta diferentes características y va de la mano con las transformaciones entre los asalariados. El principal mecanismo de baja salarial que se implementa en la Argentina es la inflación y pocas son las veces en la historia argentina que se han producido caídas nominales del salario (1995, 1996, 1999, 2000 y 2002). En general, la baja del salario se presenta en momentos inflacionarios, cuando los precios suben más rápido que los salarios. El "rodrigazo" de 1975 -con una inflación de más del $180 \%$ - constituye el punto de partida del ciclo de caída real del salario luego del estancamiento de las décadas posteriores al peronismo, descenso que se consolida pese a una breve recuperación durante la dictadura (en 1979 y 1980). Con la vuelta de la democracia en 1983, se produce una fuerte alza del salario real en los años 1983 y 1984, pero al no corresponder esta a una transformación estructural de las condiciones de compra de la fuerza de trabajo, ese incremento no se sostiene en el tiempo, incluso cuando como resultado de la apertura comercial durante la dictadura y la crisis, se produjese un proceso de quiebra de las empresas más ineficientes, acompañado de un incremento en la productividad del orden del $25,52 \%$. Pese a ser más grandes y eficientes, las empresas que operan en el país durante los ochenta siguen siendo poco competitivas. No se trata de un proceso de desindustrialización, como plantean algunos autores, sino de concentración y centralización del capital como producto de una pérdida de competitividad en el mercado mundial, dada la expansión del sudeste asiático y el menor peso de la renta diferencial como mecanismo de sostén. Por lo tanto, los capitales radicados en Argentina siguen requiriendo salarios bajos como mecanismo para compensar su ineficiencia, aunque no les alcance para potenciarse como exportadores. Por esa razón, el alza salarial no es sostenible y continúa su caída en los ochenta pese al incremento de las huelgas generales y los conflictos surgidos en el período posdictadura (IńigoCarrera, 2001; Nueva Mayoría, 2013).

Esta caída tendría su momento más agudo con la hiperinflación de 1989, cuando se observa una baja del salario real para el conjunto de la economía de más del $59 \%$. Este derrumbe se recupera en los primeros años de la presidencia de Carlos Menem (1990-1999) con un incremento que supera los niveles previos de la década de los ochenta hasta 1994, momento en el que se retomará la senda de contracción. Sin embargo, el crecimiento de los primeros ańos del menemismo no implica una mejora para el conjunto de la clase obrera, cuando se consolida una creciente población que no accede al empleo como resultado de las privatizaciones, que expulsan una parte importante de los trabajadores de las empresas hasta entonces estatales, junto al aumento de la productividad producto de la tecnificación. A la vez, se emprende un proceso de expansión del empleo no registrado -o "en negro" - y la llamada tercerización, mediante la incorporación de trabajadores fuera de convenio (el empleo no registrado alcanza prácticamente la mitad de los asalariados hacia fines de los noventa y principios del 2000).

Por otra parte, se consolida una transformación del gasto social como expresión del salario indirecto: se contrae en términos relativos el gasto destinado a salud, educación y vivienda, para dar lugar al crecimiento del componente asistencial de la política social. Se trata de aquel dirigido a las fracciones de los obreros que no logran vender su fuerza de trabajo o a quienes lo hacen en condiciones que nos les alcanza para sostener a su familia (Seiffer, Kornblihtt \& De Luca, 2012).

Hasta 1994, estas transformaciones ocurren a la par con una recuperación del salario real, pero a partir de entonces se empalman con un retorno del ciclo contractivo. La caída, que comienza en 1995, se presenta sin que medie un proceso inflacionario, sino mediante caídas del salario nominal en forma directa. Este hecho se ve posibilitado, en gran medida, por el creciente peso de la desocupación y la fragmentación, que implica la expansión del empleo no registrado y la tercerización. La llamada "flexibilización laboral", aunque no sancionada formalmente, se aplica con especial énfasis en las empresas más chicas. Esto lleva a que las empresas más grandes y con mayor control, apelen en forma creciente a la tercerización (Salvia, 2003). El menor nivel de empleo y el crecimiento de los empleos precarios, 
implican que una caída del salario real no tan pronunciada lleve a un menor ingreso de la unidad familiar, al contar con menos integrantes ocupados.

Esta situación de un salario estancado sin una fuerte caída, empezó a revertirse ante las dificultades crecientes del capital en la Argentina. La crisis de la economía, como expresión de que la deuda externa creciente no alcanza para sostener a capitalistas que se encuentran rezagados en el mercado mundial (aunque hayan invertido y aumentado su productividad) en un marco de contracción económica mundial, lleva en el 2001 a una nueva baja salarial, esta vez no de forma suave como ocurriría durante la segunda mitad de los noventa. Para efectuarla, se lleva a cabo una importante devaluación de la moneda local acompañada de una fuerte expansión de la inflación. Esta caída salarial es la antesala de una fase de expansión de la economía con aumento de la rentabilidad y de la actividad industrial, en particular a partir del 2003 (Ińigo-Carrera, 2007). Algunos autores (Novick, 2006; Palomino, 2007; Panigo \& Neffa, 2009) plantean que esta nueva tendencia implica un cambio de fase en la evolución de las condiciones de vida de los asalariados. Como veremos, los incrementos en el salario que se producen a partir de la posconvertibilidad, no logran compensar la caída provocada en el 2001, a la vez que se mantiene una estructura con un alto peso del trabajo no registrado y del cuentapropismo (Jiménez, 2011).

\section{Una recuperación que no recupera (2003-2013)}

La caída del salario real alcanza, para el conjunto de la economía, el piso más bajo de la historia en el año 2001. A partir del 2003, comienza una recuperación que durará hasta el 2009, cuando se frena. La recuperación será heterogénea para los diferentes sectores de la economía y estará marcada por un proceso de negociación permanente, a través de paritarias anuales y un alto grado de conflictividad (Nueva Mayoría, 2013). Mientras que en la década de 1980 la conflictividad expresaba un intento por frenar la baja salarial en marcha, luego del ańo 2003 se constituye en un mecanismo por medio del cual y hasta 2009, se obtienen mejoras reales. Estas, sin embargo, logran llevar el nivel salarial para el conjunto de los asalariados apenas al promedio de la década de 1990 (en el periodo 20042013, el salario promedio representa el $91 \%$ del salario del período 1990-1999).

Algunos sectores, en particular los trabajadores registrados de la industria manufacturera, llegan en el 2009 a niveles salariales por encima de los presentados en la década de 1990, e incluso cercanos a los mejores ańos de la historia argentina (ver Figura 4).

Figura 4

Salario real según sector, Argentina: 1993-2007 (en pesos de 2013)

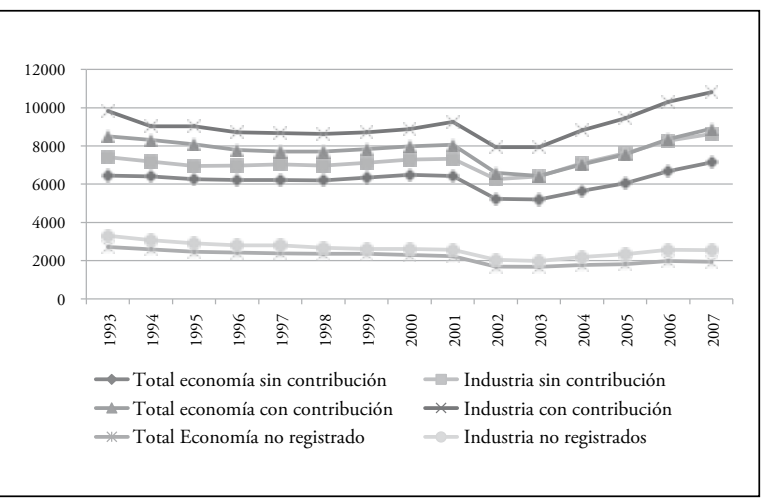

Fuente: elaboración propia en base a INDEC (cuentas nacionales, 1993-2007).

Por ejemplo, los asalariados registrados del sector manufacturero se ubican, en el 2012, con salarios reales un $2 \%$ por debajo de 1974 . Hay que tener en cuenta que esta mejora es en relación con el ingreso directo, mientras que se ha producido un empeoramiento en relación con el salario indirecto dadas una menor calidad del gasto educativo y en salud del Estado, que llevan a la necesidad de gastar una parte del salario en servicios antes cubiertos.

Esto no significa que haya disminuido el gasto social, sino que este se dirige sobre todo a quienes tienen los peores ingresos. A su vez, el sector mejor pagado dentro de los asalariados fue recargado, en los últimos años, con el pago de la cuarta categoría del impuesto a las ganancias en un porcentaje cada vez más alto, debido a la falta de actualización del mínimo no imponible y de las escalas internas del impuesto. Parte de estos impuestos sirvieron para financiar la asistencia social, en lugar de incrementar los impuestos a las ganancias de los capitalistas (Seiffer, 2012).

Con todo, esta mejora afecta a un sector minoritario de los asalariados, que vio mejorados sus ingresos en relación a los percibidos en 1990. La mayor parte se encuentra por debajo del promedio salarial pagado durante el menemismo. Esto se da porque la recuperación de la demanda laboral se hace, en su mayor parte, en las ramas de la producción con peores salarios, como son la construcción, el servicio doméstico y hotelería y restaurantes (Rodríguez-Cybulski, 2014), con un alto nivel de trabajo no registrado que se mantiene durante la década en torno al $40 \%$ (en la actualidad alcanza más del $33 \%$ ). Entre los 
sectores más damnificados, se encuentran los empleados públicos del nivel provincial y municipal, cuyo nivel salarial se encuentra por debajo del promedio del conjunto de la economía. En este pelotón están también los trabajadores agrícolas, quienes se encuentran en la rama con peores salarios pese a que al sector tuvo un crecimiento $y$ un ingreso de ganancias récord en esta década.

El aumento del empleo con persistencia de bajo salarios para la mayor parte de la población, se refleja en que por primera vez en la historia se observaron trabajadores ocupados-incluso registrados- que viven en la pobreza. La mencionada expansión de la asistencia social va dirigida, en parte, a compensar estos salarios que no alcanzan para sobrevivir. No constituye un ingreso que permite transformar la situación; solo compensarla (Seiffer, Kornblihtt $\&$ De Luca, 2012).

El dinámico crecimiento económico, combinado con altos niveles de empleo no registrado y con salarios reales que no superan los niveles previos a la década de 1990, es presentado por unos como una "tarea pendiente" del modelo, y por otros como resultado de malas políticas públicas. Lo que es ignorado por estas posturas es que una de las condiciones del fuerte crecimiento de la última década son esos bajos salarios; no se puede separar la generación de ganancias del nivel salarial. El gran crecimiento de la rentabilidad de las empresas en la última década es, parcialmente, resultado del bajo nivel salarial sostenido en la persistencia de la posibilidad de tercerizar mano de obra y de emplear trabajadores no registrados. Además, las empresas más grandes tienen salarios en blanco (y más altos) y por tanto, subcontratan mano de obra a través de empresas chicas con mayor nivel de empleo en negro y peores salarios.

A su vez, las empresas se ven beneficiadas porque parte del consumo de los asalariados es cubierto con políticas asistenciales (destinadas a quienes trabajan en negro) y los subsidios a los servicios públicos. Estas transferencias, aunque presentadas como un beneficio para el conjunto de la población, significan un abaratamiento del costo salarial para los capitalistas.

Los bajos salarios llevan a una mayor demanda de empleo que luego empuja, en parte, al alza salarial de los últimos años. Se trata de una expansión basada en capitales que, como sucede a lo largo de toda la historia argentina, tienen una productividad baja y en gran medida estancada, ya que operan con la tecnología que incorporaron en los noventa. En términos salariales, al igual de lo que pasó a mediados de los setenta, la baja del salario real fue muy fuerte, pero no llevó los costos laborales argentinos por debajo de los de Brasil ${ }^{6}$ sigue siendo una acumulación de capital sostenida con transferencias extraordinarias. A la que proviene de la baja salarial, se suma, a partir de 2003, la fuerte alza del precio de la soja que conduce la renta de la tierra a niveles récord.

A partir del 2009, el crecimiento de la renta diferencial de la tierra, junto a los bajos salarios impulsores del crecimiento de la acumulación de capital, se desacelera, lo que conduce a serios problemas en la economía. En términos de los salarios promedio para el conjunto de la economía, se observa un freno en su crecimiento y a través de un proceso inflacionario, comienza a caer el poder de consumo de los asalariados y regresa, en consecuencia, la tendencia a la caída del salario real que se presentó a mediados de 1970. En síntesis, la recuperación de los salarios para el conjunto de los asalariados hasta el 2009, apenas alcanza los niveles previos para luego estancarse y con la devaluación de fines del 2013 y principio del 2014 se agudiza la baja. A esto se suma el cepo cambiario que limita la posibilidad de ahorro de las fracciones obreras con mejores salarios. En el último año, la caída salarial en marcha-insuficiente para relanzar la acumulación-se acompaña de un aumento del desempleo (muchas veces oculto por un aumento paralelo de la inactividad por efecto desaliento), razón por la cual se presiona para ponerles límite a las paritarias y por una nueva devaluación que efectivice una baja salarial más fuerte.

\section{Conclusiones}

El análisis de la evolución del salario real del conjunto de la economía, nos permite avanzar en algunas aproximaciones. En primer lugar, la disminución de los salarios aparece, una vez más, como una fuente de compensación para la baja competitividad de las empresas locales. Es una vía por la cual el conjunto de los capitales se nutre de una fuerza de trabajo abaratada, a efectos de compensar su escasa rentabilidad. Por otra parte, la baja salarial complementa otros mecanismos utilizados por los capitales que acumulan en la Argentina, como la transferencia de renta agraria y el endeudamiento externo.

En segundo término, la tendencia decreciente del salario de los obreros argentinos comienza a manifestarse

6. Según datos del U.S. Bureau of Labor Statistics de los EE.UU. el costo laboral horario industrial en dólares en Brasil se encontraba en 2012 un $41 \%$ por debajo del argentino. 
a partir de la década de 1950 . Si bien no disminuye en términos absolutos, sí se manifiesta en términos relativos; es decir, representa un menor porcentaje del salario obtenido por los obreros de otros países. Dicho en otras palabras, el salario de los obreros argentinos deja de crecer en relación con otros países. Esto abona, en un sentido contrario, a la idea según la cual el supuesto cambio de modelo económico (de uno productivo a otro financiero) instaurado por la dictadura militar de 1976, sería el causante de la baja salarial. Lo que sí se manifiesta es la profundización de esa tendencia decreciente.

En tercer lugar, la breve recuperación salarial de los últimos años es un momento particular que no frena una tendencia de largo plazo. A nuestro juicio, se trata de una directriz sin perspectivas de freno dado que las condiciones que la generan no han cambiado. Esto se agrava por la escalada inflacionaria, que tiende a disminuir cada vez más el poder de compra de los asalariados.

El artículo deja planteado un conjunto de interrogantes que serán profundizados en futuras investigaciones. Por un lado, se tiene el análisis del salario indirecto y social, es decir, aquel que garantiza el Estado sobre la base del gasto social destinado a la cobertura de ciertos servicios de salud, educación y demás. Si bien el Estado garantiza esa parte, no paga por el capital. Surge, entonces, la pregunta acerca de la calidad de esa cobertura y quiénes la utilizan, sobre todo si se tienen en cuenta la creciente sobrepoblación relativa -manifestada en el empleo precario y sin aportes-, la desocupación abierta, los beneficiarios de planes sociales y demás.

Estos análisis, por otro lado, pueden ser profundizados con estudios sobre el salario familiar y el empleo femenino en la historia. No es casual que en el momento de mayor nivel salarial de los obreros argentinos durante la década de 1940, el empleo femenino haya sido, en términos históricos, el más bajo.

Por último, un estudio sobre la evolución del salario en Argentina amerita el análisis del nivel de ingreso en las diferentes ramas económicas, en particular en aquellas que más crecieron en las últimas décadas, como por ejemplo el empleo público, el gremio de la construcción y las ramas del comercio, hoteles y restaurantes. Se trata de los empleos que más crecieron en los últimos años y que peores salarios reciben en el conjunto de la economía.

Agradecimientos a Sebastián Cominiello y Viviana Rodríguez Cybulski, por su constante colaboración en el proyecto de investigación y por sus aportes en la lectura y revisión de este artículo.

\section{Referencias}

Altimir, O., Beccaria, L., \& Gónzalez-Rozada, M. (2002). La distribución del ingreso en Argentina, 1974-2000. Revista CEPAL 78, 55-85.

Basualdo, E. (2006). Estudios de historia económica argentina. Desde mediados del siglo XX a la actualidad. Buenos Aires: Siglo XXI.

Basualdo, E. (2009). Evolución de la economía argentina en el marco de las transformaciones de la economía internacional de las últimas décadas. En E. Arceo, \& E. Basualdo (Eds.), Los condicionantes de la crisis en América Latina. Inserción internacional y modalidad de acumulación (pp. 312-382). Buenos Aires: Clacso.

Fitzsimons, A. (2013). Producción, relaciones sociales y valor: una crítica a la teoría del patrón de acumulación basado en la valorización financiera. Razón y Revolución, 24, 85-103.

Galiani, S., \& Gerchunoff, P. (2003). The Labor Market. En G. Della Paolera \& A. Taylor (Eds.), A New Economic History of Argentina. Cambridge: Cambridge University Press.

González, M. (Diciembre, 2007). Tendencias de los salarios reales en Argentina desde 1950. Trabajo presentado en el $1^{\circ}$ Congreso Latinoamericano de Historia Económica y $4^{\circ}$ Jornadas Uruguayas de Historia Económica, Montevideo.

Graña, J. (2013). El proceso de heterogeneización y segmentación laboral como resultado del rezago productivo. Razón y Revolución, 26, 107-127.

Graña, J., \& Kennedy, D. (Diciembre, 2007). Salarios y productividad: especificidades de tamaño en la distribución del excedente. Trabajo presentado en la I Jornada de Economía Política en la Universidad Nacional de General Sarmiento, Buenos Aires- Argentina.

Iñigo-Carrera, J. (2007). La formación económica de la sociedad argentina. Volumen I. Renta agraria, ganancia industrial y deuda externa. 1882-2004. Buenos Aires: Imago Mundi.

Iñigo-Carrera, N. (Agosto, 2001). Las huelgas generales, Argentina 1983-2001: un ejercicio de periodización. Trabajo presentado en el $5^{\circ}$ Congreso Nacional de Estudios del Trabajo, Buenos Aires- Argentina.

Jiménez, M. (2011). Caracterización del trabajo independiente en Argentina. En F. Bertranou \& R. Maurizio (Eds.), Trabajadores independientes, mercado laboral e informalidad en Argentina (pp. 39-94). Buenos Aires: OIT.

Kennedy, D. (2012). Economia politica de la contabilidad social: vinculos entre la teoría de la riqueza social y sus formas de cuantificación (Tesis doctoral inédita). Universidad de Buenos Aires, Argentina.

Marshall, A. (1981). La composición del consumo de los obreros industriales de Buenos Aires, 1930-1980. Revista Desarrollo Económico 83, 351-374. 
Marx, K. (1999). El Capital. El proceso de producción del capital. México: Siglo XXI.

Novick, S. (2006). ¿Emerge un nuevo modelo económico y social? El caso argentino 2003-2006. Revista Latinoamericana de Estudios del Trabajo, 18, 53-78.

Nueva Mayoría (2013). Indicadores de conflictividad social (1980-2012). Recuperado de http://goo.gl/NY0nSf

Palomino, H. (Agosto, 2007). La instalación de un nuevo régimen de empleo en Argentina. Trabajo presentado en el 8 vo Congreso Nacional de Estudios del Trabajo, Buenos Aires-Argentina.

Panigo, D., \& J. Neffa (2009). El mercado de trabajo argentino en el nuevo modelo de desarrollo (Documento de Trabajo). Recuperado del sitio de internet del Ministerio de Economía y Finanzas Públicas, Secretaría de Política Económica: http:// goo.gl/s3M9zD

Rodríguez-Cybulski, V. (2014). Las condiciones de compraventa de la fuerza de trabajo en América Latina bajo gobiernos “progresistas”. Razón y Revolución, 27, 161-176.

Salvia, A. (Agosto, 2003). La estructura social del trabajo en Argentina: desempleo, subempleo y precariedad laboral. Trabajo presentado en el $6^{\circ}$ Congreso Nacional Estudios del Trabajo, Buenos Aires- Argentina.

Sartelli, E. (2008). Patrones en la ruta. El conflicto agrario y los enfrentamientos en el seno de la burguesía, marzo-julio de 2008. Buenos Aires: Ediciones RYR.

Seiffer, T. (2012). De nuestro bolsillo. El financiamiento obrero al Estado capitalista bajo el kirchnerismo y sus límites. El Aromo, 67, 22-23.

Seiffer, T., Kornblihtt, J., \& De Luca, R. (2012). El gasto social como contención de la población obrera sobrante en Argentina y Venezuela durante el kirchnerismo y el chavismo (2003-2010). Cuadernos de Trabajo Social, 25(1), 33-47.

Villanova, N. (2012). ¿Excluidos o incluidos? Recuperadores de materiales reciclables en Latinoamérica. Revista Mexicana de Sociología, 74(2), 245-274.

Williamson, J. (1992). The evolution of global labor markets in the first and second world since 1830: background evidence and hypotheses. (Documento de trabajo No. 36). Recuperado del sitio de internet de National Bureau of Economic Research: http://goo.gl/KsgOuJ 